\title{
Fetal akinesia deformation sequence: a case report and review of literature
}

\author{
Reena Abraham, B. Devi* \\ Department of Obstetrics \& Gynaecology, P.S.G. Institute of Medical Sciences and Research, Coimbatore, Tamil \\ Nadu, India
}

Received: 31 July 2014

Accepted: 9 August 2014

\section{*Correspondence:}

Dr. B. Devi,

E-mail: devibalasubramaniam@gmail.com

(C) 2014 Abraham R et al. This is an open-access article distributed under the terms of the Creative Commons Attribution Non-Commercial License, which permits unrestricted non-commercial use, distribution, and reproduction in any medium, provided the original work is properly cited.

\begin{abstract}
Fetal Akinesia Deformation Sequence (FADS) is a condition characterised by decreased fetal movement (fetal akinesia), multiple joint contractures (arthrogryposis), facial anomalies, intrauterine growth restriction, pulmonary hypoplasia and other developmental abnormalities. These disorders are clinically and genetically heterogenous and its etiology remains unclear. This syndrome is rare and the primary diagnosis is made when lack of mobility is noted in routine ultrasound scanning. The increasing use of ultrasound has enabled earlier detection of these cases. A 20 year old primi in her routine ultrasound at 14 weeks of gestation showed features fetal akinesia deformation sequence with increased nuchal translucency and hydrops. Early diagnosis, prenatal evaluation and better understanding of the ultrasound findings will be helpful for genetic counselling and clinical management.
\end{abstract}

Keywords: Fetal akinesia sequence, Prenatal diagnosis

\section{INTRODUCTION}

Fetal akinesia refers to a broad spectrum of disorders in which, the unifying feature is reduced or absent intrauterine fetal movements and is considered to be a lethal disorder in most cases. Associated abnormalities can include arthrogryposis (multiple joint contractures), pterygia, subcutaneous edema, fetal hydrops, pulmonary hypoplasia, craniofacial abnormalities, IUGR and other developmental abnormalities. ${ }^{1}$ FADS may be inherited in an autosomal recessive manner and it has been implied in $50 \%$ of the published cases and is listed as "rare disease" by the office of Rare diseases of the National Institute of Health (NIH). The incidence is estimated to be 1 in 12,000 reflecting causal heterogenecity. ${ }^{2}$ Various labels are given to these disorders, including fetal hypokinesia sequence, lethal Pena-Shokeir 1 syndrome, Arthrogryposis Multiplex Congenita (AMC) and multiple pterygia syndrome. ${ }^{3}$ About $30 \%$ are still born ,and the majority of those live born die of the complication of pulmonary hypoplasia. Infrequently some of the affected children may reach the age of 1 year and beyond. The increasing use of ultrasound has enabled earlier detection of these cases and the primary diagnosis made when lack of mobility and an abnormal position is noted in routine ultrasound scan. ${ }^{4}$ The syndrome is rare, about 100 cases have been described in the literature.

\section{CASE REPORT}

A 20 year old primiparous woman at 14 weeks of pregnancy, during her routine antenatal ultrasound was noted to have increased nuchal translucency, fetal akinesia with fixed flexion deformity of all four limbs due to multiple ptergium, generalised subcutaneous edema, bilateral pleural effusion, ascites, cystic hygroma and absent stomach bubble suggestive of fetal akinesia deformation sequence. There are similarities between FADS and the trisomy 18 syndrome and karyotyping permits differential diagnosis. As it is a lethal disorder she was suggested for termination of pregnancy and karyotyping of chorionic villi. Patient underwent 
termination in her home town, autopsy and karyotyping was not done due to her financial constraints.

\section{Ultrasound images}

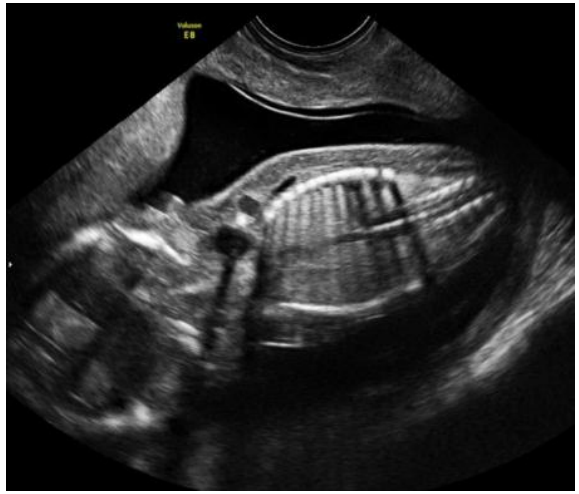

Figure 1: Subcutaneous edema.

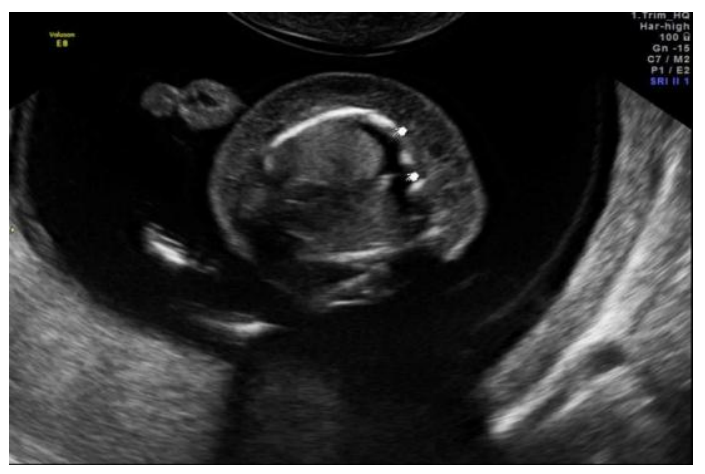

Figure 2: Bilateral pleural effusion.

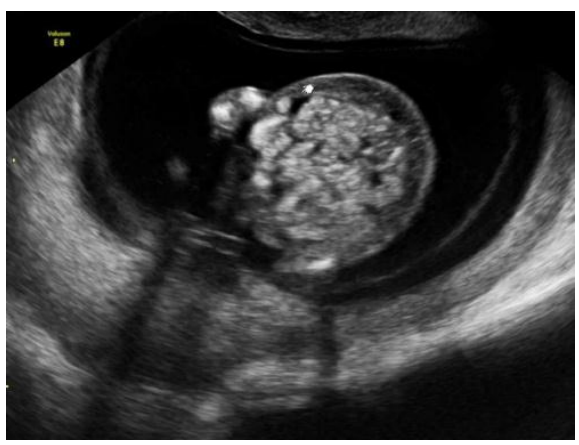

Figure 3: Ascites.

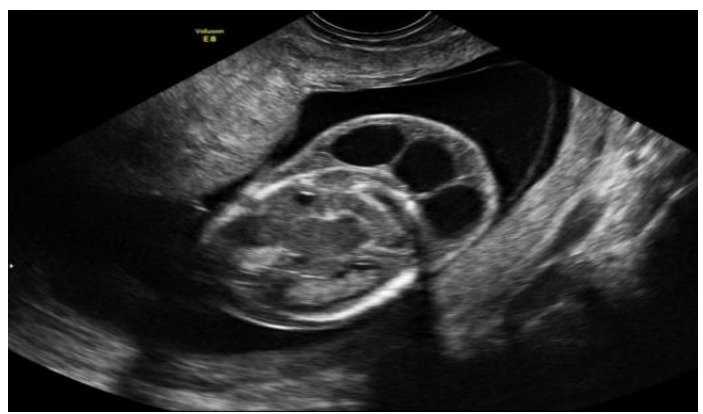

Figure 4: Cystic hygroma.

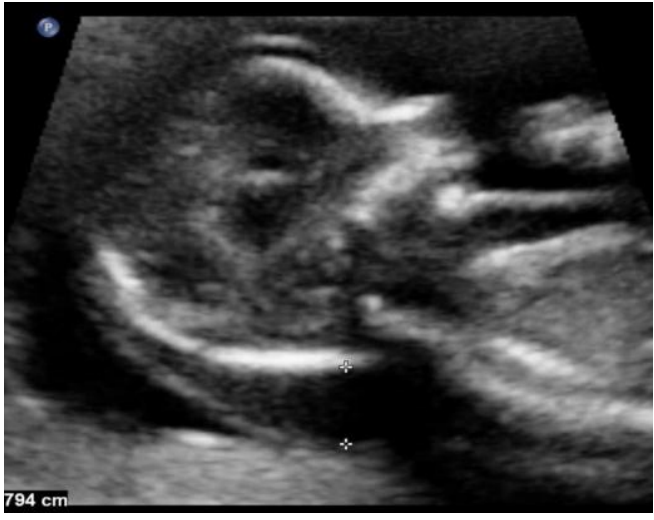

Figure 5: Increased NT.

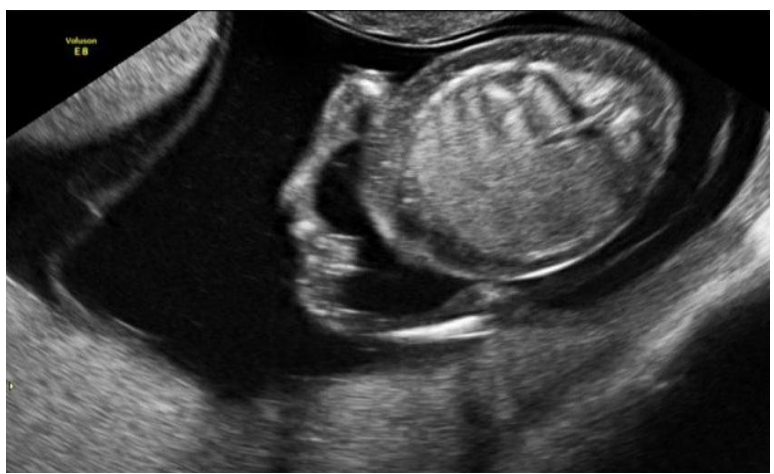

Figure 6: Absent stomach.

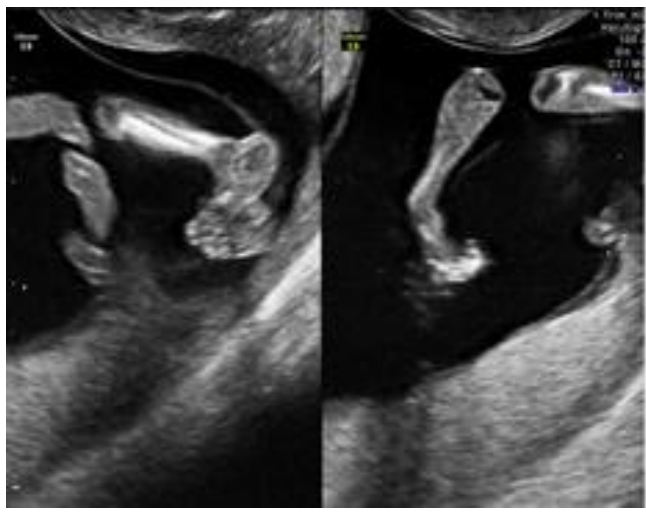

Figure 7: Fixed flexion deformity (2D).

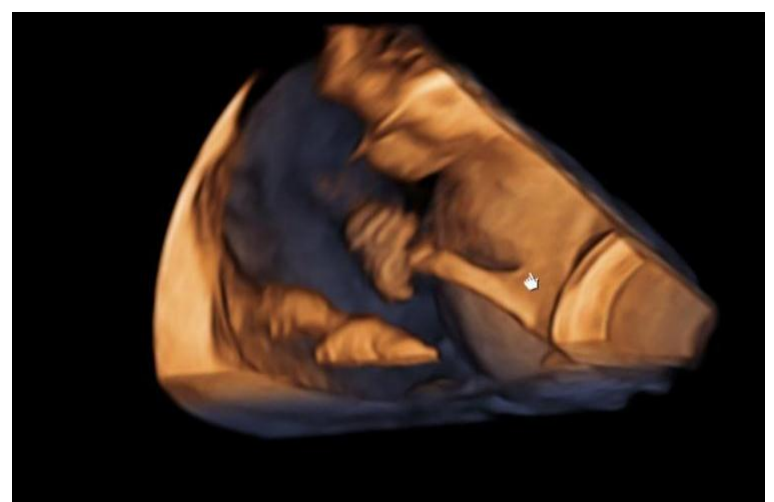

Figure 8: Fixed flexion deformity (3D). 


\section{DISCUSSION}

A fetal akinesia sequence is an event that can occur with a lack of fetal movement, these results in fetal joint contractures and pulmonary hypoplasia. Whatever the cause, the common feature of the sequence is decreased fetal activity. ${ }^{1}$ Ulnar deviation of hands, rocker - bottom feet, camptodactyly, sparse dermal ridges and absence of palmar flexion creases are the other components of the fetal akinesia sequence. Failure of normal deglutition results in polyhydramnios and lack of movement of the diaphragm and intercostal muscles leads to pulmonary hypoplasia. The lack of normal fetal movement also results in short umbilical cord and multiple joint contractures. The face appears expressionless with hypertelorism, telecanthus, poorly folded, small and posteriorly angulated ears and the mouth is small with micrognathia and high arched palate. Many of the babies are born prematurely and even when born at term, their growth is delayed and they have short neck and cryptorchidism. If they survive, they are likely to develop short gut syndrome with malabsorption.

The etiology still remains unclear but generally many causes that lead to reduced fetal movements may guide to FADS because proper fetal growth is dependent on fetal movement starting by 8 weeks of gestation. ${ }^{5,6}$ Autosomal recessive inheritance with parental consanguinity and (or) recurrence in siblings has been implied in about $50 \%$ of the published cases. The fetal akinesias are clinically and genetically heterogeneous, with causative mutations identified to date in large number of genes encoding disparate parts of the motor system. Despite recent studies identifying novel disease genes and (or) mutations (RAPSN, DOK7, CHRN 1, CHRND, CHRNG, NEB, ACTA1), the majority of fetal akinesia cases do not have genetic diagnosis. The benefits of identifying the causative mutations for each individual patient or family include accurate genetic counselling and the options of prenatal diagnosis or preimplantation genetic diagnosis. ${ }^{3}$ When pterygia are present, CHRMG, CHRNA1 and CHRND will be the order of testing. When pterygia are not evident, the order of testing should be DOK7 then RAPSN. When the presenting feature is fetal hydrops with no pterygia, testing of GLE1 should be considered.

In animal models, viruses, neuromuscular diseases, hyperthermia, limb immobilisation are responsible for contractures. ${ }^{7}$ There are multiple varied intrinsic and extrinsic causes for reduced fetal movements which includes neurological diseases (brain, spine, peripheral nerve), connective tissue defects (diastrophic dysplasia), muscle abnormalities (muscle dystrophies or mitochondrial abnormalities), space limitation within the uterus (oligohydromnios, fibroids, uterine malformations or multiple pregnancy), intrauterine or fetal vascular compromise (impaired normal development of nerves or anterior horn cell death) and maternal diseases (diabetes, multiple sclerosis, rubella, varicella, toxoplasmosis and CMV) ${ }^{8-10}$ FADS could also be a clinical manifestations of different syndromes such as dysgenesis of the nervous system observed in chromosomal abnormalities like trisomy 18 and 21. It affects approximately 1 in 2-3000 live borns ${ }^{5}$ (or) 1 in 5-10000 live births according to other authors with an approximately equal gender ratio. ${ }^{11}$

Lastly medical administration or drug abuse (curare- a skeletal muscle relaxant, misoprostol or substances such as cocaine and alcohol) during pregnancy can result in congenital contractures if given at a critical period of fetal development. $^{12,13}$

\section{Ultrasound findings and diagnosis}

Although fetal movements is observed by scan at 8 weeks gestation, most cases are diagnosed prenatally at the second or third trimester of pregnancy with ultrasound and (or) with the combination of maternal consideration for reduced fetal in utero movements. The earlier the contracture occurs or if the syndrome presents with abnormalities other than skeletal malformations, the harder the early prenatal diagnosis will be. ${ }^{14}$

Primary diagnosis is made when lack of mobility and an abnormal position is noted in routine ultrasound scanning. These findings should guide the practitioner to a careful assessment of fetal anatomy and joint. The most common detailed ultrasound findings are fixed flexion deformities, micrognathia, altered amniotic fluid volume, limb deformities, cerebral ventriculomegaly, dysmorphic features and growth retardation. ${ }^{5}$ FADS may also be present with increased nuchal translucency at 10-14 weeks ${ }^{15}$ (or) increased nuchal translucency and scoliosis at 15 weeks of pregnancy due to nuchal edema that has been found in the first and second trimester ultrasound. ${ }^{14}$ Visualisation of details of the dynamics of small anatomical structures, body and limb movements can now be done better and earlier with the use of 4D ultrasound (body and limb movements can be visualised a week earlier than with 2D) giving the possibility of diagnosis of motoric failure by the end of first trimester. ${ }^{16}$

Some of the more common signs and symptoms associated with this syndrome are abnormalities of shoulder (internal rotation), elbow (extension and pronation), wrist (volar and ulnar), and hands (flexed, abducted and externally rotated, often dislocated), knee (flexion), and foot (club foot) and in severe variety, nearly every joint is involved including jaw and back. Furthermore, complications may include respiratory problems, growth retardation, mid facial haemangioma, abdominal hernias, congenital heart defects, tracheaoesophageal fistulas and ophthalmologic abnormalities due to arthrogryposis and skeletal scoliosis. ${ }^{17}$

Diagnosis of the condition by ultrasound will depend on the gestational age and possible presence of other abnormalities, with additional MRI surveillance providing additional information such as distal muscle atrophy, abnormal muscle formation, and lung volume measurement. A combination of reduced fetal movements in utero with cystic hygroma and increased nuchal 
translucency may aid to an early diagnosis of FADS in first and (or) early second trimester of pregnancy. ${ }^{14,15}$

\section{CONCLUSION}

Better understanding of the ultrasound findings and etiology of the clinical situation offers the opportunity for careful prenatal assessment through thorough image scanning, focussing on flexion/extension of proximal and distal joints, jaw and spines.

When prenatal diagnosis is suspected family should be counselled for potential postnatal evaluation and information acquired will be helpful for family counselling concerning neonatal morbidity and mortality, labour or delivery planning and mental preparation of parents for an unfavarouble pregnancy outcome. The importance of autopsy and karyotyping should be noted to parents as it confirms prenatal diagnosis and provide families with valuable information other than that given by ultrasound and MRI scan. ${ }^{18}$

\section{Funding: No funding sources}

Conflict of interest: None declared

Ethical approval: Not required

\section{REFERENCES}

1. Chen CP. Prenatal diagnosis and genetic analysis of fetal akinesia deformation sequence and multiple pterygium syndromes associated with neuromuscular junction disorders: a review. Taiwan J Obstet Gynaecol. 2012 Mar;51(1):12-7.

2. Harold Chen. Fetal akinesia sequence. In: Harold Chen, eds. Atlas of Genetic Diagnosis and Counselling. 1st ed. New Jersey: Humana Press; 2006: 398-402.

3. Ravenscroft G, Sollis E, Charles AK, North KN, Baynam G, Laing NG. Fetal akinesia: review of the genetics of the neuromuscular causes. J Med Genet. 2011;48;793-801.

4. Kalampokas E, Kalampokas T, Sofoudis C, Deligeoroglou E, Botsis D. Diagnosing arthrogryposis multiplex congenita: a review. ISRN Obstet Gynaecol. 2012;2012:264918.

5. Navti OB, Kinning E, Vasudevan P, Barrow M, Porter H, Howarth E, et al. Review of perinatal management of arthrogryposis at a large UK teaching hospital serving a multiethnic population. Prenat Diagn. 2010;30(1):49-56.

6. Witters I, Moerman P, Fryns JP. Fetal akinesia deformation sequence: a study of 30 consecutive in utero diagnoses. Am J Med Genet. 2002;113(1):23-8.
7. B. D. Rink. Arthrogryposis: a review and approach to prenatal diagnosis. Obstet Gynaecol Surv. 2011;66(6):369-77.

8. Hall JG. Arthrogryposis multiplex congenita: etiology, genetics, classification, diagnostic approach, and general aspects. J Paediatr Orthop B. 1997;6(3):159-66.

9. Polizzi A, Huson SM, Vincent A. Teratogen update: maternal myasthenia gravis as a cause of congenital arthrogryposis. Teratology. 2000;62(5):332-41.

10. Barnes PR, Kanabar DJ, Brueton L, Newsom-Davis J, Huson SM, Mann NP, et al. Recurrent congenital arthrogryposis leading to a diagnosis of myasthenia gravis in an initially asymptomatic mother. Neuromuscul Disord. 1995;5(1):59-65.

11. Darin N, Kimber E, Kroksmark AK, Tulinius M. Multiple congenital contractures: birth prevalence, etiology, and outcome. J Paediatrics. 2002;140(1):61-7.

12. Hammond E, Donnenfeld AE. Fetal akinesia. Obstet Gynaecol Surv. 1995;50(3):240-9.

13. Coelho KE, Sarmento MF, Veiga CM, SpeckMartins CE, Safatle HP, Castro CV, et al. Misoprostol embryotoxicity: clinical evaluation of fifteen patients with arthrogryposis. Am J Med Genet. 2000;95(4):297-301.

14. 14. Scott H, Hunter A, Bedard B. Non-lethal arthrogryposis multiplex congenita presenting with cystic hygroma at 13 weeks gestational age. Prenatal Diagnosis. 1999; 19(10):966-971.

15. Hyett J, Noble P, Sebire NJ, Snijders R, Nicolaides $\mathrm{KH}$. Lethal congenital arthrogryposis presents with increased nuchal translucency at 10-14 weeks of gestation. Ultrasound Obstet Gynaecol. 1997;9(5):310-3.

16. Kurjak A, Vecek N, Hafner T, Bozek T, FundukKurjak B, Ujevic B. Prenatal diagnosis: what does four-dimensional ultrasound add? J Perinat Med. 2002;30(1):57-62.

17. Brooks JG Jr, Coster DJ. Arthrogryposis multiplex congenita: a report of two cases. Aust N Z J Ophthalmol. 1994;22(2):127-32.

18. Boyd PA, Tondi F, Hicks NR, Chamberlain PF. Autopsy after termination of pregnancy for fetal anomaly: retrospective cohort study. $\mathrm{Br}$ Med J. 2004;328(7432):137-40.

DOI: $10.5455 / 2320-1770 . i j r \operatorname{cog} 20140969$

Cite this article as: Abraham R, Devi B. Fetal akinesia deformation sequence: a case report and review of literature. Int J Reprod Contracept Obstet Gynecol 2014;3:843-6. 